\title{
RÉPERTOIRE DES DÉCOUVERTES DE LA CULTURE BODROGKERESZTUR SUR LE TERRITOIRE DE ROUMANIE
}

Sabin Adrian Luca

Le propos du présent travail est de signaler tous les points archéologiques du territoire roumain appartenant à la culture nommée. Les travaux précédents de ce genre ont donné des synthèses pour des espaces limités, à savoir: La Transylvanie ${ }^{1}$, le Banat ${ }^{2}$, le Nord-Ouest du pays ${ }^{3}$. Par rapport à ceux-ci, nous avons ramassé les informations parues plus récemment, les points enregistrés uniquement dans las répertoires locaux et les résultats de nos propres recherches ${ }^{4}$. On est ainisi parvenu à constater que - au niveau actuel des connaissances - les sites, les nécropoles, les découvertes isolées, les découvertes de métal en Roumanie représentent, du point de vue quantitatif et qualitatif, la seconde aire d'intérêt pour la culture Bodrogkerestúr, après celle de la plaine pannoniennes.

Les découvertes seront présentées suivant l'ordre alphabétiquie des localités concernées, en cherchant d'indlauer, pour les plus importeptes, les phases auxquelles elles appartiennent. Il sera également fait état des points signalés comme Bodrogkeresztúr où nos propres recherches ont infirmé l'information resue. Notons, enfin, que nos vérifications n'ont pas porté sur le seul domaine bibliographique. Jusqu'à la rédaction présente, nous avons visité sous les musées à même de nous fournir des renseignements intéressant notre sujet ${ }^{6}$.

\section{Culture Bodrogkeresztúr (cartes 1 et 2)}

1. Localité (plus Loin L) = Ampoita; lieu-dit (plus loin Id) = Peret; département (plus loin dép.) = Alba.

Description (plus loin D); Sur le manteau d'un tumulus on a trouvé un fragment céramique appartenant, à notre avis, à une phase finale de la culture, ainsi qu'une perle tubulaire de marbre.

Bibliographie (plus loin B); Ciugudean, 1991, 95, fig. 21/1.

2. $\mathbf{L}$ = Băile Herculane, Id = Peştera Hoţilor, dép. Caraş-Severin.

- D = Harmi les matériaux archéologiques publiés, il y en à qui pourraient appartenir à un horizon final de la culture?.

B = Roman 1971, 1973; Lazarovici 1975, liste 3, point 2, 25; 1983, 12.

\footnotetext{
1 Vlassa 1964.

${ }^{2}$ Lazarovici 1975; 1985.

${ }^{3}$ Németi 1988, Jercoşan 1990.

4 Revelons aussi des essais de synthèse pour l'entière aire de la culture: 100 1960, 59,60, pl. VII; Rusu 1971; Pădureanu 1982. Amplement profitables ont été pour nous les répertoires de nos collègues des différents musées, dont une mention à part mérite celui du Musée d’Arad. Nous avons également bénéficié des informations de haute importance offertes gracieusernent par le professeur dr. Mircea Rusu que nous remercions ici aussi. Nous avons effectué des reconnaissance de terrain dans les đépartements de Caraş-Severin, Timiş, Arad, Hunedoara, Alba.

${ }^{5}$ Patay 1974-1975. Il s'agit de presque 100 découvertes.

${ }^{6}$ Nous adressons nos remerciements à tous les collègues qui nous ont aidé dans le travail effectué dans les musées de TurnuSeverin, Reşiţa, Timişoara, Arad, Oradea, Carei, Satu-Mare, Baia Mare, Cluj Napoca, Alba Iulia, Deva, Sibiu, Sfântu Gheorghe, Bucarest.

${ }^{7}$ Des matériaux de ce genre se retrouvent plutoót dans le niveau I.
} 
3. $\mathbf{L}$ = Beba Veche, dép. Timiş

$\mathbf{D}=$ Cinq vases relevant de la culture ont été signalés dans la bibliographie plus ancienne.

B = Patay 1961, 93-94, fig. XXXIV/1-5; Szekely 1964, 125; Rusu, 1971, 81; Lazarovici 1975, 25, liste 2, point 3, fig. 2/9; 7/12-16; 1985, 85, 88; Pădureanu 1982, 33.

4. $\mathbf{L}$ = Blandiana, dép. Alba.

D = Les matériaux proviennent d'une station (M.Rusu) qui peut être attribuée à la phase classique de la culture.

B = Vlassa 1964, 361 (note 50 - inf. K. Horedt); Rusu, 1971, 81; Płdureanu 1982, 34.

5. $\mathbf{L}$ = Bogata de Mures, dans la proximité de la ville de Ludus, dép. Mures.

D = Une tombe a été découverte en 1903 dans une carrière de gravier.

$B=$ Roska, 1942, 157, no 84, fig. 181/2; la figure 181/1 présente, de l'avis du regretté N. Vlassa, un vase Noua; Vlassa 1964, 351, fig. 1/7; Pădureahu, 1982, 34.

6. $\mathbf{L}$ = Carei, Id = Station d'épuration de la raffinerie de sucre, dép. Satu-Mare.

D = L'inventaire appartient à une phase tardive de la culture.

B = Németi, 1988, 121-122; Iercosan 1990, 32.

7. $\mathbf{L}$ = Carmin, ld = Malu Crasnei, dép. Satu Mare.

D = Tombe isolée.

B = Németi 1988, 126; Iercoşan, 1990, 32.

8. $\mathbf{L}=$ Cămin, ld = Podu Crasnei (a et b), déd. Satu Mare.

D = Au point a - une station ${ }^{8}$, au point $\mathrm{b}$ - une nécropole?.

B = Németi 1988, 123-126; Iercoşan, 1990, 32.

9. $\mathbf{L}$ = Căpleni, Id = "A" - canton ferroviaire, dép. Satu Mare.

D = Il y a là, selon I. Németi, une station.

B = Németi 1986 - 1987, 24-26, 1988, 122; Iercoşan, 1990, 32.

10. $\mathbf{L}$ = Căpleni, Id = "H", dép. Satu Mare

D = Restes céramiques provenant - nous dit leur découvreur - d'une station.

B = Németi, 1988, 123; Iercoşan, 1990, 32.

\footnotetext{
${ }^{8}$ Németi, 1988, 123.

${ }^{9}$ Idem, 123-126.
} 
11. $L=$ Cenad $^{10}$, Id = Belo Brdo, dép. Timiş

D = L'inventaire archéologique indique la présence d'une tombe de haute époque.

B = Lazarovici 1975, 12, 14, 25, liste 1, point 11, 20, fig. 5/D1-4, 6/1-4; 1985, 25; Pădureanu 1982, 34.

12. $\mathbf{L}$ = Ciumeşti, Id = III (Fântâna păşunii?), dép. Satu Mate.

D = La collection Kovács compte plusieurs vases entiers qui paraissent appartenir à une nécropole. On a encore trouvé là deux ciseaux en cuivre. Le site archéologique se trouve à l'ouest du village, sur une colline de sable.

B = Kacsó 1969, 49-56; Rusu 1971, 81; Pădureanu 1982, 34; Iercoşan, 1990, 32.

13. $\mathbf{L}$ = Ciumeşti, ld. Râtul ţiganilor, dép. Satu Mare.

D = Les matériaux relèvent d'une station.

B = Inf. I. Németi, apırès étude de la collection Kovács de Satu Mare. Il se peut que ce soit là station que nous a signalé M.Rusu. Kacsó lui-même parle d'ailleurs d'une station: Kacsó, 1969, 52.

14. $\mathbf{L}=$ Ciumeşti, Id = Dealul cocoşat, dép. Satu Mare.

D = I. Németi porte à notre connaissance l'apparition, dans la collection Kovács, de certains matériaux attribués à l'époque.

B = Inf. I.Németi. Collection dd Musée de Satu Mare.

15. $\mathbf{L}=$ Cluj, dép. Cluj.

D = Dans un fossé de canalisation, devant le musée archéologique local, a été découverte une petite tasse ayant les caractéristiques de cette culture.

B =Roska, 1942, 135, no. 231; XOX, 1960, pl. VII; Vlassa 1964. 351, fig. 1/8.

16. $\mathbf{L}=$ Corneşti, dép. Timis.

D = Quelques fragments céramiques caractéristiques de notre culture ont été cueillis, par 0 . Radu, du territoire de la localité.

B = Lazarovici, 1975, 12, 14, 25, fig. 4/5-13; 1976, no. 15, liste 2; 1985, 85; Lazarovici Munteanu 1982, 124.

17. $\mathbf{L}=$ Corpadea, dép. Cluj.

D = Un petit case trouvé ici est attribué à cette culture.

B = Kovács, 1915, 325; Vlassa 1964, 351, fig. 3/8.

\footnotetext{
${ }^{10}$ Dans la collection Schiefmann de Timişoara, les matériaux appartenant à la culture Bodrogkeresztúr sont attribués à la localité Satu Mare (dép. d'Arad) F. Medelet nous a informé que c'est là une erreur, la véritable localité de provenance en est Cenad.
} 
18. $\mathbf{L}=$ Cristuru Secuiesc, Id = Panta cu brazi?, dép. Harghita.

$\mathbf{D}=$ Les fouilles de Benkó Elék et $Z$. Székely ont mis au jour des matériaux typiques de cette culture.

B = Székely, 1983, 31-33, fig. 4/1-2.

19. $L$ = Curtici, dép. Arad.

D = Plusieurs points énéolithiques oñte étéen enregistrés dont deux semblent appartenir à cette culture. Les fouilles systématiques entreprises ici ont été publiées par Pósta Béla.

B = Pósta Béla, 1899, 18-28; Patay 1961, 94 - 96, pl. XXXV/1-7; Székely, 1964, 125; Rusu, 1971, 81; Roman, 1971, 92; 1973, 60; Pădureanu, 1982, 33; Lazarovici, 1983, 11 (matériel inédit dans le Musée d'Arad; inf. T. Soroceanu).

20. $\mathbf{L}$ = Deva, Id = Ciangăi, dép. Hunedoara.

D = Nous penchons à croire que les matériaux relèvent d'une station Bodrogkeresztúr aux caractéristiques de la phase de transition à cette culture, bien que les observation stratigraphiques ne soient pas très claires.

B = Floca, 1950, 220-224, pl. I - XI; Székely, 1964, 125; Vlassa, 1969, 31; Kácsó, 1969, 54; Rusu, 1971, 30; Roman, 1971, 95-96; 1973, 60; Pădureanu, 1982, 33; Roman, 1978, 219.

21. $\mathbf{L}$ = Dorobanţ, Id = ancien siège de la Coopérative Agricole de Production, dép. Arad.

D = En 1960, lorsqu'on creusait la fosse d'un silo, une tombe à inhumation a été découverte où une hache à deux tranchants s'opposant en croix reposait auprès du squelette.

B = Dörner 1970, 461-462.

22. $\mathbf{L}=$ Dudeştii Vechi (ancien Beşenova Veche), Id = Bukova III, dép. Timiş

D = Il est difficile de préciser le caractère de la découverte.

B = Lazarovici, 1975, 12, 14, fig. 3/1, 3, liste 1, point 7,20.

23. $\mathbf{L}=$ Gheja, dép. Mures.

D = Dans la carrière de sable, entre Gheja et la gare de Luduş, en 1907 on a découvert une tombe.

B = Roska, 1942, 161, no 91; 162, fig. 191; Patay, 1961, 85-86, pl. XXXII/11, 13; Vlassa 1964, 352, fig. 1/9-10; Székely, 1964, 125; x x × 1960, pl. VII, Rusu 1971, 81; Pádureanu 1982, 34 (ces ouvrages continnent aussi ta bibliographie plus ancienne concernant ce problème).

24. L = Gornesti, dép. Mures.

D = Le découverte peut constituer une nécropole. 
B = Roska, 1942, 97, no. 29, fig. 122; 98, fig. 123; $\times$ × x 1960, pl. VII; Vlassa 1964, 353355, fig. 1/1-6 (avec la bibliographie ancienne pour ce point); Székely, 1964, 125; Patay 1961, 96-97, pl. XOOV/8-11; Rusu 1971, 81; Pădureanu, 1982, 34.
25. $\mathbf{L}$ = Hotoan. dép. Satu Mare.
D = Tombe isolée.
B = Németi, 1988, 126; Iercosan, 1990, 32.

26. $\mathbf{L}$ = Iliadia, ld = Funii, dép. Caraş-Severin.

D = Les fouilles dans ce point ont mis au jour un complexe qui peut s'encadrer dans la phase haute/classique de notre culture.

B = Lazarovici, 1985, 83-89; 90 - rapport ostéologique.

27. L = Iratoşu, dép. Arad.

D = L'aspect chronologique (dans le cadre de la chronologie interne de la culture) est dificile à préciser.

B = Székely, 1964, 125.

28. $\mathbf{L}$ = Luduş, Id - sur le territoire de la fabrique d'alcool, dép. Mureş.

D = La pièce que l'on attribue à notre culture est une petite tasse.

B = Roşka, 1942, 165, no 105; Vlassa 1964, 355, fig. 3/7; Pădureanu 1982, 34.

29. $\mathbf{L}$ = Mociu, dép. Cluj

D = Les matériaux semblent appartenir à une station.

B = Roska, 1942, 184, no. 247; Vlassa, 1964, 355, fig. 3/1.

30. $\mathbf{L}=$ Moldoveneşti, dép. Cluj.

D = Il s'agit d'un vase attribué à la culture Bodrogkeresztúr.

B = Roska, 1942, 299, no 40; Vlassa 1964, 355-356, fig. 2/4.

31. $\mathbf{L}=$ Mugeni, ld = Pagyvan, dép. Harghita.

D = La couche Ariujd d'ici a livrè aussi des matériaux Tisa. Cest toujours là qu'on a découvert une hache de cuivre à deux tranchats s'opposant en croix. $1975,45-67$.

B = Dumitrescu, 1968, 38; Roman, 1971, 110-111; Rusu, 1971, 80, Ferenczi et Ferenczi,

32. $\mathbf{L}=$ Ostrovu Corbului, ld = Botul Cliucului, dép. Mehedinţ. 
D = Parmi les matériaux récoltés ici, quelques-uns sont typiques de notre culture ${ }^{11}$. Ils peuvent provenir aussi de la nécropole fouillée systématiquement ici et où certaines tombes (par ex. M22) sont caractéristiques pour la culture Bodrogkeresztùr ${ }^{12}$.

$B=$ Roman, 1971, 40, fig. 2/50-58; 6-8; 34/11; 38/15; 1973, 58; 1978, 218-219, 1987, 346; Roman - Opritescu 1989, 11-38; Lazarovici 1983, 12, 1985, 85.

33. $\mathbf{L}=$ Oradea, Id = Solca II- Ghetărie, dép. Bihor.

D = Les matériaux proviennent d'une station.

B = Macrea - Crişan 1964, 356, no. 77; Rusu 1971, 81; Ignat, 1973, 11-12, 1976, 13-14; 1977, 16, 19; Iercoşan, 1990, 33.

34. $\mathbf{L}=$ Parţa, Id = tell $I$, dép. Timis.

D = Lors des fouilles dans la secţion S7 on a trouvé un fragment céramique ornementé dans le genre de notre culture.

B = Lazarovici - Munteanu 1982, 124; Lazarovici 1985, 85; Pădureanu, 1982, 33.

35. $\mathbf{L}$ = Pecica, ld = Forgaci, dép. Arad.

D = La station est à env. $5 \mathrm{~km}$ de Pecica. Les fouilles de 1989 ont dévoilé deux niveaux d'habitat appartenant à la phase haute/classique de la culture.

B = Pădureanu, 1982, 29-60; Lazarovici - Munteanu 1982, 125; Lazarovici, 1983, 11, 12, no $108 ; 1985,85,88$; Luca 1990, 89-92.

36. $\mathbf{L}=$ Pâncota, ld = La Totani, dép. Arad.

D = Il semble s'agir dans ce cas d'une station fortifiée, à en juger d'après l'aspect du terrain. On peut la dater de la haute époque de la culture.

B = Dudaş, 1970, 356.

37. Răhău, dép. Alba.

D = D'après les informations recues de $M$. Rusus on a affaire là à une station.

B = Vlassa 1964, 361/note 50 - information de K. Horedt; Rusu 1971, 81; Pădureanu, 1982, 34.

38. $\mathbf{L}=$ Reci, dép. Covasna.

D = Les trois habitations découvertes dans la station au sud de la commune, sur la rive droite de Pârâul Negru, ont été attribuées à la culture Ariudj où l'on trouve aussi des éléments Tisa.

B = Székely, 1964, 121-126; 1965, 273-274; Dumitrescu 1968, 38; Roman, 1971, 109-110; 1973, 64; Dumitrescu, 1981, 26; Roman, 1978, 219; Pădureanu 1982, 34.

\footnotetext{
"Roman, 1971, fig.7/4.

12 Roman - Opritescu, 1989 (53 tombes énélithiques).
} 
39. L = Sălacea, dép. Bihor.

D = Les informations existantes indiquent une nécropole.

B = Roska, 1942, 252, no 9; Rusu, 1971, 81; Ignat, 1973, 14, point 17.

40. $L=$ Sebes, Id = Podu Pricopului, dép. Alba.

D = Il s'agit de matériaux provenant d'une station.

B = Macrea - Crişan, 1964, anexe II, 360, no 96; Vassa, 1964, 361 (note 50 - information de K. Horedt); Rusu, 1971, 81; Pădureanu, 1982, 34.

41. $\mathbf{L}=$ Sighişel, dép. Bihor.

D = C'est là une des découvertes funéraires de la phase classique/tardive de la culture.

$B=$ Rusu, 1971, 81.

42. L = Sâniob, dép. Bihor.

D = Une tombe découverte dans les terres appartenant à la localité.

B = Roska, 1942, 266, no. 143; Patay, 1961, 97, pl. XXXVI/3-8; Székely, 1964, 125; Rusu, 1971, 81; Ignat, 1973, 15, point 20; Pădureanu, 1982, 34.

43. $\mathbf{L}$ = Sânicolaul Roman, dép. Bihor.

D = Les données existences attestent ici une nécropole.

$B=$ Rusu, 1971, 81.

44. $L$ = Sântana, Id = Holumb, dép. Arad.

D = Un des niveaux tardifs d'ici peut être attribué à la culture Bodrogkeresztúr.

B = Macrea - Crişan, 1964, 361, no 101; Rusu, 1971, 79-80; Dumitrescu, 1975, 25-32.

45. $L$ = Sânpetru German, ld = Fântâna vacilor, dép. Arad.

D = Les découvertes, fortuites et systématiques, mettent en lumière une nécropole de la phase classique.

B = Dörner, 1970, 451-455; Roman 1971, 91-92; 1973, 60; Rusu, 1971, 81; Lazarovici, 1975, 25, fig. 7/1-11; 1985, 85; Pădureanu, 1982, 34.

46. $\mathbf{L}$ = Sânpetru German, Id = Malul Înalt, dép. Arad.

D = Ici, de même qu'au lieu dit Hotarul Rech, on a trouvé une hache de cuivre à deux tranchants s'opposant en croix. Sont également rappelés quelques fragments céramiques richement ornementés, qui peuvent être attribués à notre culture.

B = Roman, 1973, 59; Roman - Németi, 1978, 12. 
47. L = Slatina Timiş, Id = Gura Ilovei (Săs), dép. Caraş-Severin.

D = L'un des groupes céramiques d'ici présente des affinités avec des matériaux typiques de la culture Bodrogkeresztùr.

B = Lazarovici - Munteanu, 1982, 121 et suiv.; groupe III-IV, 124-125; Lazarovici, 1983, 12; 1985,85 .

48. $L=$ Şiria, dép. Arad.

D = Les alentours de la localité ont livré cing fragments céramiques typiques de la culture, ainsi qu'une hache en cuivre.

B = Hampel, 1899, 428, Roska, 1942 a, 83, 1942, 305, no 76; Patay, 1961, 98, pl. XXXVI/12; Székely, 1964, 125.

49. L = Târgu Mures, dép. Mureş.

D = Les fouilles de I. Kovács en 1909 et 1910 ont mené à la découverte de complexes archéologiques où les matériaux appartenant à la culture Ariudj étaient mêlés à des éléments propres de notre culture.

B = Kovács, 1915, 232-253 (résumé aux pages 299-310); Roska, 1942, 167, no 121, fig. 198; XOX, 1960, pl. VII; Vlassa, 1964, 358-359, fig. 2/3; 3/2; Székely, 1964, 125; Dumitrescu, 1968, 37-38; Roman, 1971, 108-109; 1973, 63; Pădureanu, 1982, 34.

50. L = Unirea, dép. Alba.

D = On a de là un vase publié comme tel.

B = Roska, 1942, 92, no. 90, fig. 119; Vlassa, 1964, 359, fig. 25; Roman 1973.

51. $L$ = Vezendiu, dép. Satu Mare.

D = Station signalée par I. Németi.

B = Németi, 1988, 127-129; Iercoşan, 1990, 32.

Il existe encore d'autres points publiés, à un moment ou autre, comme étant Bodrogkerestúr, mais qui ne sauraient être ajoutés à l'énumération ci-dessus. Afin toutefois d'éviter d'éventuelles discussions à ce propos, nous donnerons ci-après leurs noms accompagnés de l'attribution culturelle proposée par nous-même ou par les auteurs qui sont revenue sur leurs opinions initiales (carte 4).

1. $L$ = Buziaş, Id = Pârpora 1, dép. Timiş.

D = Les matériaux de cet endroit sont à attribuer à la phase de transition à l'époque du bronze, non pas à notre culture.

B = Lazarovici, 1983, 11, note 108.

2. $\mathbf{L}=$ Cherestur, dép Timiş.

D = Le vase découvert ici est plutôt du type Tiszàpolgár. 
B = Lazarovici, 1975, 22, fig. 5/CI, liste 1, point 12.

3. $\mathbf{L}$ = Cicir, Id = Balastiera, dép. Arad.

D = Dans un second article, l'auteur infirme sa première attribution à notre culture des découvertes concernées.

B = Pădureanu, 1973, 399; 1982, 33.

4. $\mathbf{L}$ = Criş, dép. Hunedoara.

D = L'endroit n'est que signalé, sans d'autres précisions.

B = Székely, 1964, 125, point 4, fig. 5.

$5 . \mathbf{L}$ = Dedrad, dép. Mures.

D = Ce faciès peut être contemporain de la culture dont nous nous occupons. En l'absence d'autres données, ce point est rangé parmi ceux insuffisamment précisés.

B = Vlassa, 1969, note 6.

6. L = Măgheruş, dép. Covasna.

D = Insuffisance d'arguments.

B = Székely, 1964, 125; 1965, 273-274.

7. $\mathbf{L}=$ Sfântu Gheorghe, Id = Epestetö, dép. Covasna.

D = Nous nous rattachons à l'opinion que cette découverte peut appartenir à la culture Bodrogkeresztúr. Cependant, la précarité des arguments et le fait que nous ne sommes pas parvenu à voir les matériaux nous ont recommandé de ne pas inscrire ce point parmi les acquis sûrs.

B = Roska, 1942, 246-247, fig. 305/5; Roman, 1971, 102.

8. $\mathbf{L}=$ Şintereag, dép. Bistriţa-Năsăud.

D = Le facièes défini par Vlassa pourrait également être attribué à notre culture.

B = Vlassa, 1969, note 6 .

9. $L$ = Zăbrani, dép. Arad.

D = Comme nous n'avons pas trouvé dans les dépots du Musée d'Arad les matériaux impliqués ici, il est préférable de faire figurer cette découverte parmi les points incertains.

B = Lazarovici, 1983, 11, note 108 et 12.

10. $\mathbf{L}=$ Valea lui Mihai, dép. Bihor.

D = Il semble s'agir plutôt du domaine Tiszapolgár.

B = Rusu, 1971, 81. Peut-être qu'il se réfère aux matériaux ou au point publiés chez Ignat 1973, 17, note 27 . 
11. L = Vultureni, dép. Cluj.

$\mathbf{D}=$ On se trouve devant un aspect de mixture qui peut être contemporain de notre culture.

B = Roman, 1973, 62.

12. L = Valea Rea, dép. Hunedoara.

D = Les vases provenant de cette découverte semblent apartenir à des époques préhistoriques de plus tard.

B = Roska, 1942, 235, note 13; XXX, 1960, pl. VII; Vlassa, 1964, 357-358; Rusu, 1971, 81.

13. $\mathbf{L}=$ Ocna Sibiului, dép. Sibiu.

D = Vu le caractère unique de la pièce, on a du mal à estimer si elle appartient ou non à notre culture. Il est préférable d'attendre des découvertes futures les éclaircissements nécessaires.

B = Roska, 1942, 305, no 85; Vassa 1964, 356-357, fig. 36.

On attribue ordinairement à la culture Bodrogkeresztúr aussi les dépôts de haches en bronze comprenant les types Jászladány et Mezökeresztes ${ }^{13}$ (carte 3 ).

1. L = Beius, dép. Bihor.

D = Dépôt de haches à deux tranchants s'opposant en croix.

$B=$ Rusu, 1971, 81.

2. $\mathbf{L}$ = Cluj, dép. Cluj.

D = Dépôt de heches à deux tranchants s'opposant en croix.

B = Rusu, 1971, 81.

3. $\mathbf{L}$ = Dejani, dép. Timiş.

D = Dépôt de haches à deux tranchants s'opposant en croix.

B = Roska, 1942, 32, Patay 1974/1975, 67, point 131.

4. $\mathbf{L}$ = Gheorghieni, dép. Covasna.

D = Dépôt de haches à deux tranchants s'opposant en croix.

B = Roska, 1942, 101, fig. 126; Bakó, 1968.

5. $\mathbf{L}$ = Josani, dép. Bihor.

D = Dépôt de haches à deux tranchants s'opposant en croix.

$B=$ Rusu, $1971,81$.

${ }^{13}$ Vulpe, 1975 , fig. 1 , p. $29-31 ; 37-48$ 

6. $L=$ Tarcea, dép. Bihor.
D = Dépôt de haches à deux tranchants s'opposant en croix.
B = Rusu, 1971, 81; Patay, 1974/1975, 67, point 132.

Il est connu le fait que les découvertes de dépôts comptant des objets en or de l'époque concernée, sont généralement encadrée dans la culture Bodrogkeresztúr. Cest aussi le cas d'une découverte plus ancienne:

1. $\mathbf{L}=$ Moigrad, dép. Sălaj (carte 3).

D = Dépôt formé de plusieurs catégories d'objets en or, dont se détache la grande idole du type "en violon".

$$
\text { B = Rusu, 1971, 81; Iercosan, 1990, 33; Makkay, } 1989 .
$$

Pour que le tableau de l'époque où s'épanouit la culture Bodrogkerestúr soit complet, on notera aussi les découvertes relevant du groupe Decea Mureşului, groupe qui - à nos yeux - occupe un espace temporel contemporain de la phase débutante de notre culture (carte 5).

1. $\mathbf{L}=$ Aiud, Id = microsecteur III, dép. Alba.

D = La découverte a été faite à l'occasion des traveaux de construction d'un quartier d'habitation. La céramique provenait, nous dit l'auteur de la découverte, d'une station.

B = Ciugudean, 1978, 39-53.

2. $\mathbf{L}$ = Cetea, dép. Alba.

D = Les trois sceptres de type Decea nous portent à attribuer cette découverte à la culture discutée.

B = Opriţescu, 1978, 95.

3. $\mathbf{L}$ = Decea Mureşului, dép. Alba.

D = I. Kovács a examiné dans ce cas 15 tombes à inhumation qui ont dès le début soulevé de vife commentaires.

B = Kovács 1928-1932, 89-101; 1944, 3.21; Roska 1942, 159-160, no 89; fig. 187-190; XXX 1960, pl. VII, 54-60, 74; Vlassa 1964, 359; Rusu 1971, 82; Roman 1973, 64-65; Dâmboviţa 1978, 65; Lazarovici 1983, 11; Opritescu 1978, 87-98.

4. $\mathbf{L}=$ Feldioara, dép. Cluj.

D = A.D.Opriţescu penche à attribuer à une station les trois couteaux de type Decea et le sceptre découverts ici.

B = Opriţescu, 1978, 95. 

5. $\mathbf{L}=$ Goleşti, dép. Mureş.
D = Un sceptre.
B = Opritescu, 1978, 95.

6. $L$ = Ludus, dép. Mures.

D = Une station appartenant à notre groupe nous a été signalée dans la proximité de la ville.

$$
\text { B = Informaţion M. Rusu. }
$$

7. $\mathbf{L}$ = Mescreac, dép. Alba.

D = Une station

B = Rusu, 1971, 82.

8. $\mathbf{L}$ = Mirăslău, dép. Alba.

D = La station se trouve à environ $0,5 \mathrm{Km}$. de la nécropole de Decea Mureşului, sur une haute terrasse.

B = Kovács, 1928-1932, 98; Rusu, 1971, 82.

9. $\mathbf{L}$ = Sântana, ld = Holumb, dép. Arad.

D = Un vase indique la présence du groupe Decea.

B = Dumitrescu, 1975, 25.32.

10. $\mathbf{L}$ = Sântioana de Mureş, dép. Mures.

D = A l'occasion d'une périégèse faite sur les terres appartenant à cette localité M. Rusu a découvert une station qui pourrait être attribuée au groupe Decea.

B = Information M. Rusu.

11. $\mathbf{L}=$ Şard, dép. Alba.

D = Lors de l'excavation d'un puits on a découvert un sceptre de type Decea.

B = Information I.Al. Aldea. La pièce se trouve au Musée d'Alba Iulia.

12. $\mathbf{L}=$ Ocna Sibiului, dép. Sibiu.

D = Il s'agit d'un sceptre de type Decea. Nous ignorons quel est le rapport entre cette pièce et le vase à couvercle inscrit ci-dessus parmi les découvertes Bodrogkeresztúr incertaines (position 13).

$\mathbf{B}=$ Roska, 1942, 305, no. 85; Opriţescu, 1978, 95.

Espérons que ce travail, avec les illustrations annexés, permettra de se faire une image sur la culture Bodrogkeresztúr en Roumanie, en tant que partie intégrante des phénomènes de l'énéolithique final dans le Sud-Est européen. 